Cita: Adell, F. L.; Castillo, I.; Álvarez, O.; Tomás, I. (2019). Valores personales en el baloncesto de formación y su relación con el bienestar y la intención futura de práctica a través de la motivación. Cuadernos de Psicología del Deporte, Vol 19(2), 227-242

\title{
Valores personales en el baloncesto de formación y su relación con el bienestar y la intención futura de práctica a través de la motivación
}

\author{
Personal values in youth basketball and its relationship with well-being \\ and future intention to practice through motivation
}

\section{Valores pessoais no treino de basquetebol e a sua relação com o bem-estar e a intenção futura de praticar através da motivação}

\author{
Adell, F. L., Castillo, I., Álvarez, O., Tomás, I. \\ Universitat de València, Valencia, España
}

\begin{abstract}
RESUMEN
Tomando como marco teórico la teoría de los valores humanos (Schwartz, 1992; Schwartz et al., 2012), y la teoría de la autodeterminación (Deci y Ryan, 1985, 2000), el objetivo del estudio consistió en poner a prueba un modelo, en una muestra de 115 jugadores jóvenes de baloncesto $\left(M_{\text {edad }}=13.98, D T=0.13\right)$, en el que se hipotetizó que los diferentes tipos de motivación (motivación autónoma, controlada y no motivación) actuarían como mediadores de la relación entre los valores informados por los jugadores (auto-trascendencia, conservación, auto-promoción y apertura al cambio) y los indicadores de bienestar (vitalidad subjetiva y afectos positivos), así como con la intención futura de seguir practicando baloncesto. Los resultados confirmaron el efecto indirecto de los valores de autopromoción sobre la percepción de vitalidad de los jugadores, sus afectos positivos y su intención de seguir jugando en el futuro, a través de la motivación autónoma. Sin embargo, los otros tipos de motivación (motivación controlada y no motivación) no mediaron la relación entre el resto de valores y los indicadores de bienestar, ni con la intención futura de práctica deportiva. Estos resultados indican que para favorecer una mayor experiencia de bienestar en el baloncesto habría que evitar poner énfasis en valores centrados en lo individual, en el logro personal y en el dominio sobre los demás (auto-promoción), ya que la vitalidad subjetiva, los afectos positivos y la intención de seguir jugando se pueden ver reducidos, a través de una menor motivación autónoma.
\end{abstract}

Palabras clave: Valores, motivación, afectos, vitalidad, intención conductual.

\begin{abstract}
Within the framework of the theory of human values (Schwartz, 1992; Schwartz et al, 2012), and the selfdetermination theory (Deci and Ryan, 1985, 2000), the aim of the study was to test a model, in a sample of 115 young basketball players $\left(M_{\text {age }}=13.98, S D=0.13\right)$, which hypothesized that different types of motivation (autonomous motivation, controlled motivation and non-motivation) would act as mediators in the relationship between the players' values (self-transcendence, conservation, self-enhancement and openness to change) and two indicators of well-being (subjective vitality and positive affects), as well as of the future intention to continue
\end{abstract}

Correspondence to: Isabel Castillo. Facultad de Psicología. Av. Blasco Ibañez, 21.

46010 Valencia. Tel: +34963864577. Email: Isabel.Castillo@uv.es 


\section{Adell, F. L., Castillo, I., Álvarez, O., Tomás, I.}

practicing basketball. The results supported the indirect effect of self-promotion values on the players' perception of subjective vitality, their positive affects and their intention to continue playing in the future, through autonomous motivation. However, the other types of motivation (controlled motivation and amotivation) did not mediate the relationship between the other three values and the indicators of well-being, nor with the future intention of sport practice. These results indicate that to generate a greater experience of well-being in basketball it should be avoided placing emphasis on values centred on the individual, on personal achievement and on mastery over others (selfpromotion), since subjective vitality, positive affects and the intention to continue playing could be reduced, through the decrease of autonomous motivation.

Keywords: Values, motivation, affects, subjective vitality, intentions.

\section{RESUMO}

Baseado na teoria dos valores humanos (Schwartz, 1992; Schwartz et al, 2012) e na teoria da autodeterminação (Deci e Ryan, 1985, 2000), o objetivo do estudo foi testar um modelo de relação em 115 jovens jogadores de basquetebol $\left(M_{\text {idade }}=13.98, D T=0.13\right)$, em que se hipotetizou que os diferentes tipos de motivação (motivação autónoma, motivação controlada e não motivação) actuariam como mediadores da relação entre os valores dos jogadores (autotranscendência, conservação, autopromoção e abertura à mudança) e os indicadores de bem-estar (vitalidade subjectiva e afecções positivas), bem como da intenção futura de continuar a praticar basquetebol. Os resultados confirmaram o efeito indirecto dos valores de autopromoção na percepção de vitalidade dos jogadores, nos seus afectos positivos e na sua intenção de continuar a jogar no futuro, através de uma motivação autónoma. No entanto, os outros tipos de motivação (motivação controlada e não motivação) não mediaram a relação entre o resto dos valores e os indicadores de bem-estar, nem com a intenção futura da prática desportiva. Estes resultados indicam que, para favorecer uma maior experiência de bem-estar no basquetebol, seria necessário evitar colocar ênfase nos valores centrados no indivíduo, na realização pessoal e no domínio dos outros (autopromoção), uma vez que a vitalidade subjectiva, os afectos positivos e a intenção de continuar jogando poderiam ser reduzidos, através de uma motivação menos autónoma.

Palavras chave: Valores, motivação, afeto, vitalidade, intenção comportamental.

\section{INTRODUCCIÓN}

La práctica deportiva de los más jóvenes se ha convertido en una actividad de gran interés psicosocial, tanto por la capacidad que se le supone de transformar el carácter a partir de la transmisión de valores positivos (Côté, Strachan, y FraserThomas, 2008; Whitehead, Telfer, y Lambert, 2013), como por ser una actividad que promueve el bienestar tanto físico como psicológico (WHO, 2011). En el contexto deportivo, una de las variables que ha sido relacionada directamente con el bienestar experimentado con el deportista, es la motivación. En concreto, se ha demostrado que estar autónomamente motivado está asociado a mayores niveles de funcionamiento positivo y a un mejor ajuste personal (Balaguer, Castillo, y Duda, 2008; Ryan y Deci, 2002, 2017). Además, estudios previos realizados en el marco del baloncesto de formación, evidencian que los valores personales también se expresan a través de las conductas, como son las conductas de liderazgo del entrenador (Castillo, Adell, y Álvarez, 2018), y de las actitudes (prosociales o antisociales) durante el juego (Adell, Castillo, y Álvarez, en prensa). Estos estudios han puesto de manifiesto la importancia de identificar los valores humanos de base para potenciar conductas y actitudes más adaptativas durante la práctica del baloncesto.

Los valores son metas deseables, estables a través de las situaciones y del tiempo, que varían en importancia, y que sirven como principios guía en la vida de las personas (Rokeach, 1973; Schwartz, 1992; Schwartz et al., 2012). Los valores se van a diferenciar entre ellos por el tipo de motivación que expresan, señalando aquello que nos importa en la 


\section{Valores, motivación, bienestar e intención futura en baloncesto formación}

vida a cada uno de nosotros, para la posterior organización consciente de nuestras emociones, actitudes y conductas. En su teoría de valores, Schwartz (1992) realizó una propuesta de clasificación de los valores que probablemente haya sido la más influyente hasta la fecha en la literatura científica sobre el tema, en concreto su teoría define 10 valores básicos: universalismo, benevolencia, tradición, conformidad, seguridad, poder, logro, hedonismo, estimulación y autodirección. En 2012 refinó su teoría (Schwartz et al., 2012), subdividiendo algunos de los valores básicos (i.e., universalismo, benevolencia, conformidad, seguridad, poder y autodirección), y añadiendo los valores humildad y apariencia, completando así los 19 valores básicos de su actual formulación (para su definición véase Tabla 1).

Los valores de la teoría refinada de Schwartz et al. (2012) se representan en forma de estructura circular (véase Figura 1) donde los valores que están próximos en la estructura serían valores compatibles entre sí (e.g., logro y poder), mientras que los valores opuestos en la estructura entrarían en conflicto (e.g., logro y universalismo). Estos 19 valores se organizan en dos dimensiones bipolares de orden superior que resumen las relaciones entre los valores básicos. Estas dimensiones son Auto-trascendencia (preocupación por el bienestar de los demás) versus Autopromoción (preocupación por uno mismo); y Conservación (tendencia a preservar el orden y la tradición) versus Apertura al Cambio (tendencia a exponerse a novedades y cambios). La autotrascendencia y la conservación (a excepción de la seguridad personal) centran el foco en lo social, mientras que los valores de apertura al cambio $\mathrm{y}$ autopromoción centran el foco en lo individual. Además, los valores en sí mismos poseen un enfoque de crecimiento, libre de ansiedad o de autoprotección y evitación de la ansiedad. Los valores de autotrascendencia, de apertura al cambio y en parte el valor de logro, son considerados valores de crecimiento, libres de ansiedad; mientras que los valores de autopromoción y los valores de conservación son considerados valores de autoprotección y de evitación de la ansiedad (véase Figura 1). Esta estructura es universal, y se sugiere que se mantendrá invariante independientemente de que unas personas tengan unas prioridades de valor diferente a las que puedan tener otras personas (Bardi y Schwartz, 2003).

Tabla 1. Definición de los 19 valores en la teoría refinada de Schwartz (traducido de Schwartz et al., 2012).

\begin{tabular}{|c|c|}
\hline $\begin{array}{l}\text { Dimensiones y } \\
\text { valores básicos }\end{array}$ & Definiciones conceptuales \\
\hline \multicolumn{2}{|l|}{ Auto-trascendencia } \\
\hline $\begin{array}{l}\text { Universalismo- } \\
\text { preocupación }\end{array}$ & $\begin{array}{l}\text { Compromiso con la igualdad, la } \\
\text { justicia y la protección de las } \\
\text { personas }\end{array}$ \\
\hline Universalismo-naturaleza & $\begin{array}{l}\text { Preservación del medio ambiente } \\
\text { natural }\end{array}$ \\
\hline Universalismo-tolerancia & $\begin{array}{l}\text { Aceptación y comprensión de los } \\
\text { que son diferentes de uno mismo }\end{array}$ \\
\hline Benevolencia-confianza & $\begin{array}{l}\text { Ser un miembro confiable y de } \\
\text { confianza del grupo }\end{array}$ \\
\hline Benevolencia-cuidado & $\begin{array}{l}\text { Devoción por el bienestar de los } \\
\text { miembros del grupo }\end{array}$ \\
\hline \multicolumn{2}{|l|}{ Conservación } \\
\hline Tradición & $\begin{array}{l}\text { Mantener y preservar las } \\
\text { tradiciones culturales, familiares } \\
\text { y religiosas }\end{array}$ \\
\hline Humildad & $\begin{array}{l}\text { Reconocer la insignificancia de } \\
\text { uno en el esquema más amplio } \\
\text { de las cosas }\end{array}$ \\
\hline Conformidad-reglas & $\begin{array}{l}\text { Cumplimiento de normas, leyes } \\
\text { y obligaciones formales }\end{array}$ \\
\hline Conformidad-interpersonal & $\begin{array}{l}\text { Evitar molestar o dañar a otras } \\
\text { personas }\end{array}$ \\
\hline Seguridad-personal & $\begin{array}{l}\text { Seguridad en el entorno } \\
\text { inmediato }\end{array}$ \\
\hline Seguridad-social & $\begin{array}{l}\text { Seguridad y estabilidad en la } \\
\text { sociedad en general }\end{array}$ \\
\hline Apariencia & $\begin{array}{l}\text { Seguridad y poder a través de } \\
\text { mantener la imagen pública y } \\
\text { evitar la humillación }\end{array}$ \\
\hline \multicolumn{2}{|l|}{ Autopromoción } \\
\hline $\begin{array}{l}\text { Poder- } \\
\text { dominancia }\end{array}$ & $\begin{array}{l}\text { Poder a través de ejercer control } \\
\text { sobre las personas }\end{array}$ \\
\hline Poder-recursos & $\begin{array}{l}\text { Poder a través del control de los } \\
\text { recursos materiales y sociales }\end{array}$ \\
\hline Logro & $\begin{array}{l}\text { Éxito según los estándares } \\
\text { sociales }\end{array}$ \\
\hline \multicolumn{2}{|l|}{ Apertura al cambio } \\
\hline Hedonismo & Placer y gratificación sensual \\
\hline Estimulación & Entusiasmo, novedad y cambio \\
\hline $\begin{array}{l}\text { Auto-dirección } \\
\text { pensamiento }\end{array}$ & $\begin{array}{l}\text { Libertad para cultivar las propias } \\
\text { ideas y habilidades }\end{array}$ \\
\hline Auto-dirección acción & $\begin{array}{l}\text { Libertad para determinar las } \\
\text { propias acciones }\end{array}$ \\
\hline
\end{tabular}

Nota. Las dimensiones de los valores básicos aparecen en cursiva y negrilla. 


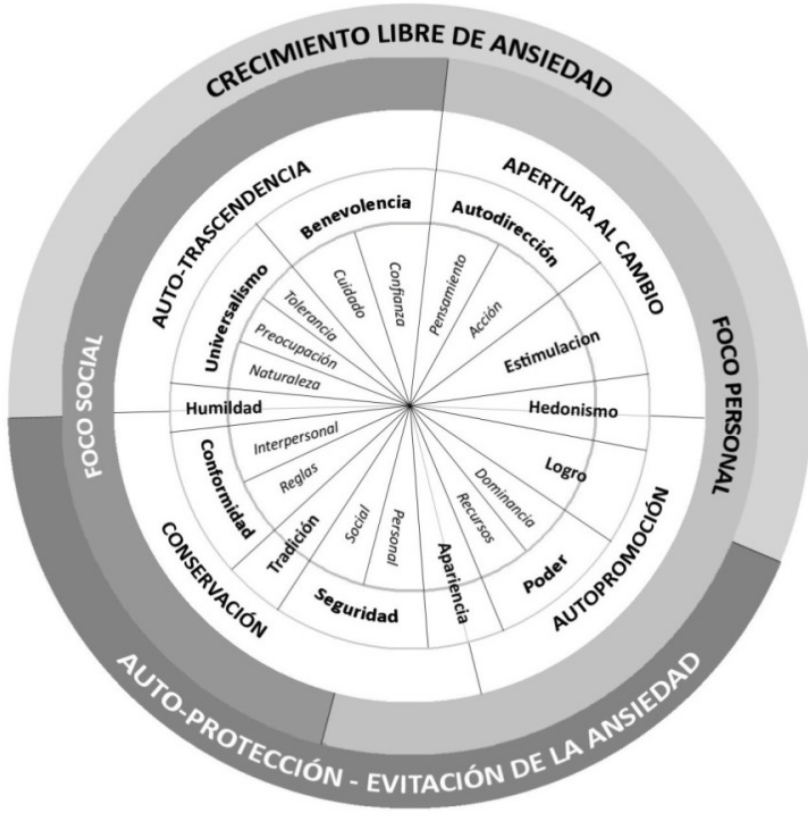

Figura 1. Estructura circular de los 19 valores básicos de Schwartz. Traducida y adaptada de Schwartz et al. (2012)

Para Sortheix y Schwartz (2017), las características foco en lo social versus foco en lo individual, por una parte, y crecimiento versus autoprotección, por otra, son las que hacen que pueda existir una relación entre los valores y el bienestar. Los valores con características de autoprotección reducirán el bienestar porque motivan la subordinación de la persona hacia expectativas impuestas socialmente (conservación), o buscan controlar y dominar a otras personas (autopromoción) para controlar la ansiedad. Por el contrario, los valores de apertura al cambio generarán bienestar porque motivan la autorrealización individual, el disfrute y la libre expresión de ideas, mientras que los valores de autotrascendencia generarán bienestar por la motivación individual que supone alcanzar el bienestar de los demás.

Siguiendo a Schwartz y Sortheix (2018), la relación entre los valores y el bienestar puede plantearse desde tres enfoques diferentes: 1) porque el contenido de los valores en sí mismo es lo que genera bienestar o malestar (relación directa) (e.g., Sagiv y Schwartz, 2000; Sortheix y Schwartz, 2017); 2) porque los valores del contexto pueden ser más o menos congruentes con los valores del individuo, y este grado de congruencia genera un determinado grado de bienestar (e.g., Sagiv y Schwarzt, 2000; Sortheix y Lönnqvist, 2015); o 3) porque las características del entorno facilitan o dificultan la consecución de metas personales (expresadas en valores), generando bienestar o malestar respectivamente (e.g., Oishii, Diener, Suh, y Lucas, 1999). Entendiendo el entorno como elemento clave de la motivación personal (Deci y Ryan, 1985; Ryan y Deci, 2000), en esta investigación nos planteamos la relación entre valores y bienestar desde el tercer punto señalado.

Una de las teorías motivacionales más relevantes que hace propuestas teóricas relacionadas con el crecimiento positivo y el bienestar de las personas es la Teoría de la Autodeterminación (SDT; Deci y Ryan, 1985; Ryan y Deci, 2000). Según la SDT, las personas pueden estar motivadas cuando realizan conductas que reflejan sus valores, creencias e intereses (motivación autónoma o autodeterminada). Además, pueden estar motivadas por influencia de fuerzas externas que les llevan a la realización de las conductas por coerción o presión, sin tener en cuenta sus valores y creencias (motivación controlada). Por último, las personas pueden no tener un propósito por el que participar en una actividad (no motivación). Tanto la motivación autónoma como la controlada, son constructos multidimensionales que incorporan diferentes tipos de regulación. La motivación autónoma estaría formada por la motivación intrínseca, la regulación integrada y la regulación identificada, mientras que la motivación controlada estaría formada por la regulación introyectada y la regulación externa. La motivación intrínseca representa la tendencia de las personas a realizar una actividad porque es en sí misma agradable y placentera, siendo la forma más autónoma de motivación. La satisfacción experimentada es inherente a la actividad. La regulación integrada se refiere a cuando la actividad que se realiza es completamente congruente con los valores, o metas de la persona (e.g., juego a baloncesto porque es una forma de expresar quién soy yo). La regulación identificada se refiere a que la regulación de la conducta se produce por aspectos que son personalmente importantes para el individuo (e.g., juego a baloncesto porque valoro los beneficios de este deporte). La regulación introyectada se refiere a 


\section{Valores, motivación, bienestar e intención futura en baloncesto formación}

conductas reguladas por presiones externas que la persona ha interiorizado (e.g., juego a baloncesto porque me sentiría culpable si lo dejara). Finalmente, la regulación externa describe conductas que son reguladas por contingencias externas al individuo (e.g., dinero o amenazas).

Se han encontrado varios paralelismos entre la SDT y la investigación desarrollada en valores (Kasser, 2002; Schwartz y Sortheix, 2018). Para la SDT, el bienestar experimentado por una persona se vincula con la satisfacción de tres necesidades psicológicas básicas: competencia, autonomía y relación con los demás (Deci y Ryan, 1985; Ryan y Deci, 2000). Dado que los valores derivarían de las necesidades del individuo (Kasser, 2002), la necesidad de competencia se vincularía con el valor de logro, la necesidad de autonomía con el valor de autodirección, y la necesidad de relación con los valores de universalismo y benevolencia (Schwartz y Sortheix, 2018). Siendo así, si como confirma la investigación existe relación entre la satisfacción de las necesidades psicológicas básicas y un mayor nivel de motivación autodeterminada (e.g., Balaguer et al., 2008), sería lógico encontrar una alta relación entre los valores de benevolencia, universalismo y autodirección con un mayor grado de motivación autodeterminada. Lo mismo sucede con la motivación controlada, si está relacionada con la necesidad de las personas de obtener aprobación y admiración social, y de evitar la censura social, parece lógico que este tipo de motivación debería estar relacionada con los valores de seguridad, conformidad, poder y tradición.

Balaguer, Castillo, Quested y Duda (2013) estudiaron la relación entre los valores personales de deportistas adolescentes con su regulación motivacional, encontrando que la motivación intrínseca se relacionó positivamente con el universalismo y la benevolencia, y negativamente con el poder y con el logro. La regulación introyectada se relacionó positivamente con la estimulación. La regulación externa se asoció positivamente con la tradición, con el poder y con la estimulación. Por último, la no motivación se relacionó positivamente con el poder. Si nos centramos en los factores de segundo orden de la teoría de Schwartz, la auto-trascendencia se relacionó positivamente con la motivación intrínseca y negativamente con la regulación introyectada y con la externa; mientras que la autopromoción se relacionó positivamente con la regulación externa y la no motivación, y negativamente con la motivación intrínseca. La dimensión apertura al cambio vs conservación no arrojó resultados estadísticamente significativos en cuanto a su relación con las regulaciones motivacionales de los deportistas adolescentes.

La investigación en el contexto físico-deportivo, ofrece evidencias que asocian la motivación autónoma a un funcionamiento psicológico óptimo, con consecuencias deseables para la salud y el bienestar (Balaguer et al., 2008; Balaguer, Castillo, Duda, y García-Merita, 2011; López-Walle et al., 2011), o la intención de continuar practicando deporte (Balaguer, Castillo, Duda, Quested, y Morales, 2011); mientras que la motivación controlada se relacionaría con respuestas cognitivas, afectivas o conductuales más negativas como por ejemplo el burnout, una menor intención de práctica futura (Balaguer, Castillo, Duda, Quested, et al., 2011), o una mayor ansiedad (Pineda-Espejel, LópezWalle, y Tomás, 2015).

El bienestar se refiere a la experiencia completa y funcionamiento óptimo del ser humano (Ryan y Deci, 2001). Se puede abordar desde dos perspectivas diferentes: la hedónica, o bienestar entendido como la búsqueda del placer y la evitación del sufrimiento, y la eudamónica, referida a la perspectiva que se tiene de los logros que se han conseguido en la vida, y cuánto de satisfecho está el individuo con lo realizado. En este estudio, vamos a utilizar dos indicadores de bienestar: la vitalidad subjetiva y los afectos positivos.

La vitalidad subjetiva es un indicador eudaimónico, definido como la experiencia psicológica de estar lleno de entusiasmo, de ánimo, y de sentirse con mucha energía (Ryan y Frederick, 1997). Las investigaciones realizadas dejan evidencia sobre el efecto positivo que tienen tanto el apoyo a la autonomía que ejercen los entrenadores (González et al., 2016; López-Walle et al., 2012), como la motivación intrínseca de los deportistas (Balaguer, Castillo, Cuevas, y Atienza, 2018), sobre la vitalidad subjetiva.

Los afectos positivos son indicadores de bienestar hedónico. Reflejan el grado en que una persona se 
siente entusiasta, activa y alerta (Watson, Clark, y Tellegen, 1988). La investigación previa relaciona los niveles más altos de motivación autodeterminada con los afectos positivos (Barbosa-Luna, Tristán, Tomás, González, y López-Walle, 2017).

Por último, algunos investigadores han estudiado la relación existente entre las regulaciones motivacionales y la intención conductual, dado el valor que esta última tiene como predictor de la conducta (Ajzen y Fishbein, 1980). En este sentido, investigaciones previas indican que la motivación autónoma predice positivamente la intención de práctica futura, mientras que la motivación controlada y la no motivación la predicen de manera negativa (e.g., Balaguer, Castillo, Duda, Quested, et al., 2011; Ntoumanis, 2001).

El presente estudio tuvo como objetivo analizar la relación de los valores personales de los jóvenes jugadores de baloncesto con el bienestar que estos experimentan, así como en su intención futura de seguir jugando a baloncesto, estudiando la motivación del jugador como mediador en esta relación. De acuerdo con la literatura previa que ha analizado estas relaciones, se hipotetiza que los valores personales de auto-trascendencia y de apertura al cambio estarán relacionados positivamente con la motivación autónoma de los deportistas y negativamente con la motivación controlada. Por su parte, los valores personales de autopromoción y de conservación estarán relacionados positivamente con la motivación controlada y la no motivación, y negativamente con la motivación autónoma. A su vez, la motivación autónoma se relacionará positivamente con la vitalidad subjetiva, los afectos positivos, y la intención futura de práctica; mientras que la motivación controlada y la no motivación se relacionarán con estos aspectos (vitalidad subjetiva, afectos positivos e intención futura de práctica) de forma negativa.

Hasta la fecha, son pocos los trabajos que han abordado la relación existente entre los valores personales y el bienestar en el contexto deportivo, y aún menos, estudios que hayan abordado dicha relación en el ámbito específico del baloncesto. El presente trabajo se convierte, por tanto, en el primero que aborda la relación entre los valores personales, el tipo de motivación y el bienestar experimentado por los jugadores practicantes de baloncesto, en el contexto de formación de dicho deporte.

\section{MATERIAL Y MÉTODOS}

\section{Participantes}

La muestra de estudio estuvo compuesta por 115 jugadores de baloncesto pertenecientes a 10 clubes españoles de baloncesto. Todos los deportistas eran de nacionalidad española, hombres, de categoría cadete y tenían 13 o 14 años $(M=13.98, D T=0.13)$ en el momento de la administración de los instrumentos. Los jugadores empezaron a jugar al baloncesto entre los 4 y los 12 años $(M=6.43 ; D T=$ 2.28), e informaron de una media de 3.64 años $(D T=$ 2.72) en su actual club.

\section{Instrumentos}

Valores personales. Los valores personales se midieron con los 57 ítems de la versión española del Portrait Values Questionnaire - Revised (PVQ-R; Schwartz et al., 2012). Cada uno de los 19 valores de la Teoría Refinada de los Valores Humanos propuesta por Schwartz, viene representado por tres ítems, y se pregunta al deportista sobre cuánto considera que él se parece a una persona que viene descrita en cada ítem en términos de preferencias de valores. A su vez estos 19 valores se agrupan en cuatro dimensiones de orden superior (autotrascendencia, conservación, autopromoción, y apertura al cambio). El jugador debe responder en una escala que va desde el 1 (No se parece en absoluto a mi) hasta el 6 (Muy parecido a mi). Un ejemplo de ítem sería 'Es importante para él buscar siempre diferentes cosas que hacer'. Existe evidencia de la validez y fiabilidad del instrumento en investigaciones previas (Castillo et al., 2018; Cieciuch et al., 2014; Schwartz et al., 2012). Para la realización del estudio se calcularon y utilizaron las puntuaciones en las dimensiones de orden superior (para más detalle véase Figura 1).

Tipos de motivación. La motivación durante la práctica del baloncesto se evaluó con los 20 ítems de la versión española (Viladrich et al., 2013) del Cuestionario de Regulación Conductual en el Deporte (BRSQ; Lonsdale, Hodge, y Rose, 2008). El cuestionario se compone de cinco subescalas con cuatro ítems cada una: motivación intrínseca, regulación identificada, regulación introyectada, 


\section{Valores, motivación, bienestar e intención futura en baloncesto formación}

regulación externa y no motivación. Este cuestionario no recoge la dimensión de regulación integrada ya que siguiendo lo señalado por Vallerand (1997) este tipo de regulación no resulta prevalente hasta la adultez. El deportista debe responder en una escala que oscila desde el 1 (Muy en desacuerdo) hasta el 5 (Muy de acuerdo). Los ítems del cuestionario vienen encabezados por la frase 'Juego a baloncesto en este equipo' y el deportista debe contestar sobre los motivos por los que juega a baloncesto. De acuerdo con las indicaciones sugeridas por la SDT (Deci y Ryan, 1985) se combina los ítems de las subescalas motivación intrínseca y regulación identificada para crear la variable Motivación Autónoma (e.g., 'Porque me gusta'), y los ítems de las subescalas regulación introyectada y regulación externa para crear la variable Motivación Controlada (e.g., 'Para satisfacer a las personas que quieren que lo practique'). Se mantiene la variable No motivación, en la que un ejemplo de ítem sería 'Aunque me pregunto por qué estoy jugando a este deporte'. Investigaciones previas confirman la validez y fiabilidad de la escala con deportistas (Balaguer et al., 2011; Viladrich et al., 2013).

Vitalidad subjetiva. La vitalidad subjetiva se evaluó con los seis ítems de la versión española (Castillo, Tomás, y Balaguer, 2017) de la Escala de Vitalidad Subjetiva (SVS, Ryan y Frederick, 1997). Esta escala evalúa cuánta energía y viveza siente el deportista, a través de una escala que oscila desde el 1 (No es verdad) hasta el 7 (Verdadero). Un ejemplo de ítem es 'Me siento vivo y vital'. La fiabilidad y validez de la escala ha sido puesta de manifiesto en investigaciones previas (Castillo et al., 2017; González, Castillo, García-Merita, y Balaguer, 2015).

Afectos positivos. Los afectos positivos se midieron usando la dimensión de afectos positivos del Cuestionario de Afectos Positivos y Negativos (PANAS; Watson, Clark, y Tellegen, 1988). Los jugadores contestaron el grado en el que habían experimentado cada una de las 10 emociones en las últimas semanas en una escala del $1(\mathrm{Nada})$ al 5 (Extremadamente). Un ejemplo de ítem sería 'emocionado'. La fiabilidad de la escala en estudios previos ha sido satisfactoria (Bartholomew, Ntoumanis, Ryan, Bosch, y Thøgersen-Ntoumani, 2011; González et al., 2015).
Intención futura de práctica. Se utilizó la versión española (Balaguer, Castillo, Duda et al., 2011) de la Escala de Intención Futura de Práctica (Chatzisarantis, Biddle, y Meek, 1997). Esta escala consta de 4 ítems, que los jugadores contestan en una escala que oscila desde el 1 (No tiene nada que ver conmigo) hasta el 7 (Se ajusta totalmente a mi). Un ejemplo de ítem sería 'Intentaré seguir jugando a baloncesto la próxima temporada'. La fiabilidad del instrumento se ha confirmado en investigaciones previas (Álvarez et al., 2012; Balaguer et al., 2011; Castillo et al., 2010).

\section{Procedimiento}

La Comisión de Ética en Investigación Experimental de la Universidad de Valencia dio su aprobación para llevar a cabo el estudio, donde participaron los 10 equipos de baloncesto provenientes de toda España, que competían en un torneo nacional. Tras conseguir la aprobación de la dirección técnica del torneo para poder encuestar a los participantes, se contactó con los directores de cantera de los diferentes Clubes para explicarles telefónicamente los objetivos de la investigación y conseguir su aprobación para hablar con los entrenadores. Una vez que éstos aprobaron la acción, el siguiente paso fue explicar mediante carta tanto a los entrenadores como a los padres de los deportistas los objetivos de la investigación, y que dieran su consentimiento para que el jugador participase en el estudio. Los deportistas, también fueron informados por carta y verbalmente sobre cuáles era los objetivos, y que se necesitaba su aprobación para continuar con la investigación.

Los jugadores de los equipos participantes, cumplimentaron los cuestionarios entre el 14 y 16 de diciembre de 2017, en una hora que fue acordada entre el investigador responsable de recoger los datos y los entrenadores responsables de los equipos, para que no distorsionase ni los horarios de competición ni los horarios de descanso, en una sala habilitada para tal efecto en el hotel donde los equipos estaban alojados, y en un tiempo aproximado de 25 minutos. Previo a la recolección de los datos, se recordó a los participantes que el tratamiento de los datos garantiza el anonimato y la confidencialidad, señalando que la participación era voluntaria, así como la importancia de ser sincero y honesto con las respuestas que se facilitaban. 
Adell, F. L., Castillo, I., Álvarez, O., Tomás, I.

\section{Análisis estadístico}

Antes de proceder al análisis de datos, se examinaron los datos faltantes así como los valores atípicos univariados. No se encontraron datos atípicos y los datos faltantes del estudio estuvieron por debajo del $0.5 \%$, por lo que no representa un problema para los análisis (e.g., Graham y Hofer, 2000). Se realizaron análisis descriptivos y de correlaciones, así como análisis de consistencia interna (alfa de Cronbach) de los instrumentos utilizados para el estudio. Estos análisis se llevaron a cabo mediante el paquete estadístico SPSS 21.

Se pusieron a prueba cuatro modelos diferentes, mediante path análisis, utilizando el programa Mplus versión 6.12 , y el método de estimación de máxima verosimilitud. En cada uno de estos modelos se utilizó como variable predictora uno de los cuatro valores, como mediadoras los tres tipos de motivación (motivación autónoma, motivación controlada y no motivación), y como variables dependientes los dos indicadores de bienestar (vitalidad subjetiva y afectos positivos) así como la intención futura de práctica. Tal como se ha indicado, teniendo en cuenta el número de parámetros en los modelos propuestos, se utilizaron las medias como indicadores de cada una de las variables, es decir, se rodaron modelos de path análisis utilizando variables observadas. Para determinar el ajuste de los modelos se consideraron la raíz del promedio del error de aproximación (RMSEA), la raíz del promedio residual estandarizado (SRMR), el índice de TuckerLewis (TLI), y el índice de ajuste comparativo (CFI). Valores de RMSEA y de SRMR por debajo de .08 se consideran óptimos, y valores de TLI y CFI superiores a .90 indican un ajuste aceptable de los datos. Finalmente, para estimar los efectos indirectos (EI) testados en los modelos, utilizamos el intervalo de confianza (IC) bootstrap corregido de sesgo, tal como aparece implementado en Mplus (Lau y Cheung, 2012). Este método permite obtener una estimación del efecto indirecto (como el producto de los coeficientes de regresión implicados en la cadena de mediación), y un intervalo de confianza al 95\% (IC 95\%) para dicho efecto indirecto. Si el intervalo de confianza no incluye el valor cero, se confirma el efecto indirecto evaluado.

\section{RESULTADOS}

Estadisticos descriptivos, fiabilidades y correlaciones entre variables

Los estadísticos descriptivos, así como las fiabilidades, se muestran en la Tabla 2. Los deportistas informaron tener como valores prioritarios la apertura al cambio, la autotrascendencia y la conservación, encontrándose las puntuaciones por encima de la media escalar, mientras que en el valor de autopromoción se obtuvo la puntuación más baja, por debajo de la media escalar. Los deportistas informaron tener una alta motivación autónoma, así como sentirse vitales, con afectos positivos y con intención de seguir practicando baloncesto en el futuro. Las fiabilidades fueron adecuadas para todas las escalas y subescalas del estudio, con valores alfa de Cronbach que oscilaron entre .75 y .86 .

Tabla 2. Descriptivos y fiabilidades de las variables del estudio

\begin{tabular}{lcccr}
\hline & Rango & Media & $D T$ & Alfa \\
\hline Auto-trascendencia & $1-6$ & 4.75 & 0.39 & .82 \\
Conservación & $1-6$ & 4.23 & 0.32 & .86 \\
Autopromoción & $1-6$ & 3.28 & 0.76 & .81 \\
Apertura al cambio & $1-6$ & 4.82 & 0.40 & .76 \\
\hline Motivación autónoma & $1-5$ & 4.48 & 0.46 & .76 \\
Motivación controlada & $1-5$ & 2.15 & 0.69 & .75 \\
No motivación & $1-5$ & 1.62 & 0.89 & .86 \\
\hline Vitalidad subjetiva & $1-7$ & 5.27 & 1.03 & .83 \\
Afectos positivos & $1-5$ & 3.81 & 0.61 & .82 \\
Intención práctica futura & $1-7$ & 6.53 & 0.79 & .82 \\
\hline
\end{tabular}

Nota. Las medias y desviaciones típicas de los valores reflejan las prioridades de los valores y se basan en centrar las respuestas de cada jugador en torno a su media para los 57 items y luego sumar la media general (4.34) para todos los encuestados en la misma escala para restaurar la escala original. Las alfas se computan utilizando las respuestas sin centrar.

El análisis de correlaciones mostró que las variables que se relacionan de forma significativa lo hacen en la dirección esperada. En concreto, el valor de autotrascendencia se asoció de manera negativa y significativa con la motivación controlada y la no motivación; mientras que el valor de autopromoción se relacionó de forma negativa y significativa con la motivación autónoma $\mathrm{y}$ de forma positiva $\mathrm{y}$ significativa con la motivación controlada y con la no motivación. La motivación autónoma se relacionó de 


\section{Valores, motivación, bienestar e intención futura en baloncesto formación}

forma positiva y significativa con los dos indicadores de bienestar (vitalidad subjetiva y afectos positivos) y con la intención de seguir jugando al baloncesto en el futuro; mientras que la no motivación se asoció de manera negativa y significativa con las tres variables mencionadas (vitalidad subjetiva, afectos positivos, e intención futura de práctica) (véase Tabla 3 al final del manuscrito).

\section{Modelos de path análisis}

Los índices de bondad de ajuste de los cuatro modelos puestos a prueba aparecen en la Tabla 4, mostrando todos ellos un buen ajuste a los datos. Atendiendo a la estimación de los parámetros de los modelos (véase Tabla 5 al final del manuscrito), los resultados indican que el valor de auto-trascendencia predijo de manera negativa y significativa la motivación controlada y la no motivación; mientras que el valor de autopromoción predijo de manera negativa la motivación autónoma, y de manera positiva la motivación controlada y la no motivación. Por su parte, la motivación autónoma predijo positivamente la vitalidad subjetiva, los afectos positivos y la intención de práctica futura; mientras que la no motivación predijo negativamente la vitalidad y la intención futura de práctica. Por último, respecto a los efectos indirectos (EI), los resultados confirmaron que el valor de autopromoción se asocia de manera negativa y significativa con la vitalidad subjetiva (EI $=-.16$; IC $95 \%=[-.29,-.02])$, los afectos negativos (EI $=-.08$; IC 95\% $=[-.15,-.01])$, y la intención de práctica futura $(\mathrm{EI}=-.07$; IC $95 \%=$ $[-.15,-.01])$, a través de la motivación autónoma.

Tabla 4. Índices de bondad de ajuste de los modelos de path análisis puestos a prueba

\begin{tabular}{lcccccc}
\hline Modelo & $\chi^{2}$ & gl & RMSEA & CFI & TLI & SRMR \\
\hline Auto-trascendencia & 2.35 & 3 & .00 & 1.00 & 1.02 & .02 \\
Conservación & 5.89 & 3 & .09 & 0.97 & 0.90 & .04 \\
Autopromoción & 2.35 & 3 & .00 & 1.00 & 1.02 & .02 \\
Apertura al cambio & 1.34 & 3 & .00 & 1.00 & 1.06 & .01 \\
\hline
\end{tabular}

Nota. Para todos los valores de $\chi 2, p>.05 ; \mathrm{gl}=$ grados de libertad; RMSEA = raíz del promedio del error de aproximación; $C F I=$ indice de ajuste comparativo; $T L I=$ indice Tucker-Lewis; SRMR = raiz del promedio residual estandarizado.
Los cuatro modelos puestos a prueba explicaron el mismo porcentaje de varianza de cada una de las variables dependientes (véase Tabla 5 al final del manuscrito). Concretamente, explicaron el 36\% de la varianza de la vitalidad subjetiva, el $19 \%$ de la varianza de los afectos positivos y el $23 \%$ de la varianza de la intención futura de seguir practicando baloncesto.

\section{DISCUSIÓN}

Tomando como marco teórico la teoría refinada de los valores humanos (Schwartz et al., 2012), y la teoría de la autodeterminación (SDT; Deci y Ryan, 1985, 2000), el objetivo de este estudio consistió en poner a prueba un modelo en jugadores jóvenes de baloncesto, en el que se hipotetizaba que los valores de auto-trascendencia y de apertura al cambio de los jugadores se asociarían a patrones motivacionales más autónomos, mientras que los valores de autopromoción y conservación se asociaría a patrones motivacionales más controlados, y a la no motivación. Se esperaba también que los deportistas autónomamente motivados informaran de una mayor vitalidad subjetiva, mayores afectos positivos $\mathrm{y}$ mayor intención futura de práctica que aquellos jugadores motivados de forma controlada, o no motivados. Finalmente, se hipotetizó que los diferentes tipos de motivación actuarían como mediadores de la relación entre los valores de los jugadores y los indicadores de bienestar, así como de la intención futura de seguir practicando baloncesto. Los resultados obtenidos han ofrecido apoyo parcial a las hipótesis planteadas en el modelo.

Los resultados de los análisis de correlación indicaron relaciones significativas entre algunos de los valores y el bienestar. En concreto, se encontraron dos relaciones significativas, mostrando que aquellos jugadores de baloncesto cuyos valores están centrados en la autopromoción personal y el logro, experimentan una menor vitalidad subjetiva; mientras que los deportistas con valores de conservación, interesados en el orden y resistentes a los cambios, experimentan mayor vitalidad. Los resultados que se ofrecen en la literatura científica sobre esta cuestión son dispares. Así, mientras que Sortheix y Lönnqvist (2015) no encuentran ningún tipo de asociación directa entre los valores de segundo orden y el bienestar, Sortheix y Schwartz (2017) sí que encuentran relación positiva entre los valores de auto- 


\section{Adell, F. L., Castillo, I., Álvarez, O., Tomás, I.}

trascendencia y apertura al cambio con el bienestar, y relación negativa entre los valores de autopromoción y los valores de conservación con el bienestar. En un reciente estudio con adultos no vinculados al deporte, Bojanowska y Piotrowsky (2018) encontraron una relación positiva entre los valores de autotrascendencia, los valores de conservación y los valores de apertura al cambio con el bienestar; mientras que los valores de autopromoción se relacionaron de forma negativa con el bienestar. Los resultados de nuestro estudio están en consonancia con los de Sortheix y Schwartz (2017) y Bojanowska y Piotrowsky (2018). No obstante, debemos ser cautos en la comparación con los estudios previos, puesto que estos autores utilizan indicadores de bienestar (e.g., satisfacción con la vida, afectos depresivos), y características muestrales diferentes (e.g., estudiantes universitarios, adultos). Así, este trabajo profundiza en la relación entre los valores y el bienestar teniendo en cuenta otros indicadores (vitalidad subjetiva $\mathrm{y}$ afectos positivos) en una muestra específica de deportistas adolescentes en etapa de formación. Siguiendo lo sugerido por Schwartz y Sortheix (2018) sobre la identificación de valores saludables y no saludables, nuestros resultados encuentran apoyo a la identificación de los valores de autopromoción como valor 'no saludable', y el de conservación como valor 'saludable'. Futuros estudios deberían indagar sobre la modulación del contexto social en la relación entre los valores y el bienestar, ya que, por ejemplo, se ha sugerido que el contexto social determina la relación entre los valores y las conductas exhibidas por los entrenadores (Castillo et al., 2018).

En lo que respecta a la relación entre los valores y la intención o deseos de continuar jugando a baloncesto en el futuro, los resultados muestran que los deportistas cuyos valores se centran en el bienestar de los demás (valores de auto-trascendencia) informan de mayor intención de seguir practicando baloncesto en el futuro, mientras que lo contrario ocurre con los deportistas centrados en la autopromoción, ya que éstos informan de una menor intención de seguir practicando baloncesto. En definitiva, estos resultados empíricos siguen apoyando lo no saludable del valor de autopromoción y apoyaría la consideración de los valores de auto-trascendencia, es decir aquellos centrados en el bienestar de los demás, como valores saludables, lo que nos ofrece información muy valiosa a nivel aplicado sobre qué tipo de valores son los que contribuyen a desarrollar mayores deseos de continuar practicando baloncesto.

Los resultados del modelo puesto a prueba muestran que cuando los jugadores de baloncesto tienen como prioritarios valores de auto-trascendencia, éstos informan de menores niveles de motivación controlada y de no motivación cuando juegan a baloncesto. Por el contrario, cuando los jugadores de baloncesto tienen valores de base relacionados con la autopromoción, informan de menores niveles de motivación autónoma y mayores niveles de motivación controlada y no motivación. Estos resultados concuerdan en parte con los obtenidos en el estudio de Balaguer et al. (2013), y apoyan las similitudes teóricas entre la SDT y la teoría de los valores humanos (Kasser, 2002; Schwartz y Sortheix, 2018). Así pues, cuando los jugadores de baloncesto priorizan la búsqueda del propio interés, el éxito personal, y el dominio sobre los demás (valores de autopromoción), su regulación motivacional será menos autodeterminada y su falta de motivación será mayor; mientras que cuando los jugadores priorizan el interés por el bienestar de los demás (valores de auto-trascendencia), se verán reducidos sus niveles de motivación controlada y de no motivación. Al igual que en el trabajo de Balaguer et al. (2013), la dimensión conservación versus apertura al cambio no parece estar relacionada con aspectos motivacionales en el deporte.

De acuerdo con los postulados de la SDT (Deci y Ryan, 1985, 2000) y en línea con la investigación previa, que informa que aquellas actividades que se realizan por factores intrínsecos conllevan mayor satisfacción y bienestar que aquellas que son realizadas por factores extrínsecos (Deci y Ryan, 2008; Ryan, Sheldon, Kasser, y Deci, 1996), los resultados han mostrado que la motivación autónoma se asocia positivamente con la vitalidad subjetiva, los afectos positivos y la intención futura de práctica; mientras que la no motivación de los jugadores de baloncesto se asocia negativamente con la vitalidad subjetiva, y la intención de seguir practicando en el futuro. De este modo, los jugadores de baloncesto que practican baloncesto por factores intrínsecos como el crecimiento personal o mantener una relación satisfactoria con el resto de compañeros, se sienten más vitales, tienen una afectividad más 


\section{Valores, motivación, bienestar e intención futura en baloncesto formación}

positiva, y quieren en mayor medida seguir practicando baloncesto. Por otra parte, aquellos jugadores de baloncesto que no están motivados para la práctica, se sienten con menos vitalidad, y desean en menor medida seguir jugando. Estos resultados van en la línea de investigaciones previas realizadas sobre las regulaciones motivacionales y diferentes indicadores de bienestar en el contexto deportivo (Álvarez et al., 2009; Balaguer et al., 2008, 2011; Barbosa-Luna et al., 2017; Chatzisarantis et al. 1997; López Walle et al., 2011) y en el contexto de la educación física (Granero-Gallegos, BaenaExtremera, Sánchez-Fuentes, y Martínez-Molina, 2014; Ntoumanis, 2001). El estar motivado de forma autónoma se relaciona con los indicadores de bienestar estudiados, así como con la intención futura de seguir practicando baloncesto. Tal evidencia, indica la importancia que tiene que los jugadores de baloncesto desarrollen altos niveles de motivación autodeterminada para tener una experiencia más positiva de la práctica del baloncesto, así como aumentar su intención de seguir siendo físicamente activo en el futuro.

Por último, los resultados de la presente investigación ofrecen evidencia empírica respecto al papel mediador de la motivación en la relación entre los valores de autopromoción y el bienestar, así como entre la autopromoción y la intención futura de práctica. Concretamente, los valores de autopromoción reducen la percepción de vitalidad de los jugadores, sus afectos positivos y su intención de seguir jugando en el futuro, a través del efecto que ejercen sobre la motivación autónoma. Sin embargo, no encontramos que los tipos de motivación medien la relación de los valores de auto-trascendencia, los valores de conservación, y los valores de apertura al cambio, con los indicadores de bienestar utilizados en el presente estudio, así como con la intención futura de práctica deportiva. Estos resultados están informando sobre el error que supone poner énfasis en valores centrados en lo individual, en el logro personal y en el dominio sobre los demás, ya que la vitalidad subjetiva, los afectos positivos y la intención de seguir jugando se van a ver reducidos por dichos valores de autopromoción, de forma indirecta a través de una reducción de la motivación autónoma. La no confirmación del papel mediador de la motivación entre algunos valores y los efectos positivos de la práctica de baloncesto, nos sugiere considerar los valores como antecedentes directos de la experiencia positiva de la práctica actual e intención de práctica de baloncesto en jóvenes jugadores.

El presente estudio tiene sus limitaciones ya que se trata de un estudio correlacional por lo que no podemos establecer causalidad en las relaciones. Asimismo, es posible que la muestra compuesta por jugadores adolescentes todos ellos hombres, esté limitando la variabilidad en los valores, así como la relación entre valores, motivación y bienestar, por lo que puede limitar la generalizabilidad de los resultados a otros deportes o contextos deportivos. No obstante, tales resultados permiten sugerir futuros estudios longitudinales, centrados en muestras compuestas de hombres y mujeres, así como de diferentes deportes y edades, que nos permitan avanzar en el conocimiento de las relaciones entre los valores, la motivación y las consecuencias que estas variables tienen a nivel emocional, cognitivo y conductual.

\section{APLICACIONES PRÁCTICAS}

Los resultados de este estudio sugieren aplicaciones prácticas que pueden ser de utilidad para las diferentes figuras de autoridad y referencia (e.g., entrenadores, directores deportivos, padres y madres) de los jóvenes que practican baloncesto. Es recomendable que estas figuras fomenten la práctica del baloncesto por motivos intrínsecos (como la diversión, la mejora personal o la buena relación con sus compañeros), más que por motivos extrínsecos (como la victoria o el dominio sobre los rivales), con el objetivo de que los adolescentes experimenten una mayor vitalidad, mayores afectos positivos y una mayor adherencia a la práctica del baloncesto en el futuro. Además, deberán tener en cuenta que el fomento de valores de autopromoción poniendo el foco en el dominio sobre los rivales o en el logro de resultados, típicos del deporte profesional más que del deporte de formación, conllevará una reducción del bienestar experimentado por los jóvenes jugadores, ya que éstos generarán que se sientan menos motivados autónomamente, reduciendo la posibilidad de experiencias positivas de la práctica deportiva y aumentando la posibilidad de que el jugador abandone la práctica deportiva. 


\section{REFERENCIAS}

1. Adell, F.L., Castillo, I., y Álvarez, O. (en prensa). Personal and sport values, goal orientations and moral attitudes in youth basketball players. Revista de Psicología del Deporte.

2. Ajzen, I., y Fishbein, M. (1980). Understanding attitudes and predicting social behavior. New Jersey, NJ: Prentice-Hall, Inc.

3. Álvarez, M. S., Balaguer, I., Castillo, I., y Duda, J. L. (2009). Coach autonomy support and quality of sport engagement in young soccer players. The Spanish Journal of Psychology, 12(1), $\quad 128 \quad-\quad 138 . \quad$ doi: $10.1017 / \mathrm{S} 1138741600001554$

4. Álvarez, M. S., Balaguer, I., Castillo, I., y Duda, J. L. (2012). The coach-created motivational climate, young athletes' well-being and intentions to continue participation. Journal of Clinical Sport Psychology, 6(2), 166-179. Disponible en: http://citeseerx.ist.psu.edu/viewdoc/download?do $\mathrm{i}=10.1 .1 .469 .4578 \& \mathrm{rep}=$ rep $1 \&$ type $=$ pdf

5. Balaguer, I., Castillo, I., y Duda, J. L. (2008). Apoyo a la autonomía, satisfacción de las necesidades, motivación y bienestar en deportistas de competición: un análisis desde la teoría de la autodeterminación. Revista de Psicología del Deporte, 17(1), 123-139. Disponible en: https://www.rpdonline.com/article/view/246

6. Balaguer, I., Castillo, I., Cuevas, R., y Atienza, F. (2018). The importance of coaches' autonomy support in the leisure experience and well-being of young footballers. Frontiers in Psychology. 9, 840. doi:10.3389/fpsyg.2018.00840

7. Balaguer, I., Castillo, I., Duda, J. L., Quested, E., y Morales, V. (2011). Predictores sociocontextuales y motivacionales de la intención de continuar participando: Un análisis desde la SDT en danza. Revista Internacional de Ciencias del Deporte, 25(7), 305-319. doi: 10.5232/ricyde2011.02505

8. Balaguer, I., Castillo, I., Duda, J. L., y GarcíaMerita, M. (2011). Asociaciones entre la percepción del clima motivacional creado por el entrenador, orientaciones disposicionales de meta, regulaciones motivacionales y vitalidad subjetiva en jóvenes jugadores de tenis. Revista de Psicología del Deporte, 20(1), 133-148. Disponible en: https://www.rpdonline.com/article/view/851

9. Balaguer, I., Castillo, I., Quested, E., y Duda, J. L. (2013). How do values relate to motivation? En J. Whitehead, H. Telfer, y J. Lambert (Eds.), Values in youth sport and physical education (pp. 119-133). London: Routledge. doi:10.4324/9780203114155

10. Barbosa-Luna, A. E., Tristán, J. L., Tomás, I., González, A., y López-Walle, J. M. (2017). Climas motivacionales, motivación autodeterminada, afectos y burnout en deportistas: enfoque multinivel. Acción Psicológica, 14(1), 105-117. Disponible en: http://scielo.isciii.es/pdf/acp/v14n1/1578-908Xacp-14-01-00105.pdf

11. Bardi, A., y Schwartz, S. H. (2003). Values and behavior: Strength and structure of relations. Personality and Social Psychology Bulletin, 29, 1207-1220. doi:10.1177/0146167203254602

12. Bartholomew, K. J., Ntoumanis, N., Ryan, R., Bosch, J. A., y Thøgersen-Ntoumani, C. (2011). Self-determination theory and diminished functioning: The role of interpersonal control and psychological need thwarting. Personality and Social Psychology Bulletin, 37, 1459-1473. doi:10.1177/0146167211413125

13. Bojanowska, A., y Piotrowski, K. (2018). Two levels of personality: temperament and values and their effects on hedonic and eudaimonic well-being. Current Psychology, 1-9. Disponible en:

https://link.springer.com/article/10.1007/s12144018-0043-0

14. Castillo, I., Adell, F. L., y Álvarez, O. (2018). Relationships between personal values and leadership behaviors in basketball coaches. Frontiers in Psychology, 9, 1661. doi:10.3389/fpsyg.2018.01661.

15. Castillo, I., Falcó, C., Álvarez, O., Morales, V., Duda, J. L., y Balaguer, I. (2010). Relationships between passion, motivational regulations and well-being in vocational dancers. 4th International SDT Conference. Ghent (Belgium). 


\section{Valores, motivación, bienestar e intención futura en baloncesto formación}

16. Castillo, I., Tomás, I., y Balaguer, I. (2017). The Spanish-version of the Subjective Vitality Scale: Psychometric properties and evidence of validity. The Spanish Journal of Psychology, 20, e26, 1-8. doi:10.1017/sjp.2017.22

17. Chatzisarantis, N. L., Biddle, S. J., y Meek, G. A. (1997). A self- determination theory approach to the study of intentions and the intention behavior relationship in children's physical activity. British Journal of Health Psychology, 2(4), 343-360. Disponible en: http://selfdeterminationtheory.org/authors/nikoschatzisarantis/

18. Cieciuch, J., Davidov, E., Vecchione, M., y Schwartz, S. H. (2014). A hierarchical structure of basic human values in a third-order confirmatory factor analysis. Swiss Journal of Psychology, 73, 177-182. doi:10.1024/14210185/a000134

19. Côté, J., Strachan, L., y Fraser-Thomas, J. (2008). Participation, personal development, and performance through youth sport. En N. Holt (Ed.), Positive youth development through sport (pp. 34-45). New York, NY: Routledge

20. Deci, E. L., y Ryan, R. M. (1985). Intrinsic motivation and self-determination in human behavior. New York, NY: Plenum Press. doi:10.1007/978-1-4899-2271-7

21. Deci, E. L., y Ryan, R. M. (2000). The 'what' and 'why' of goal pursuits: Human needs and the self-determination of behavior. Psychological Inquiry, $\quad 11, \quad 227-268$. doi:10.1207/S15327965PLI1104_01

22. Deci, E. L., y Ryan, R. M. (2008). Selfdetermination theory: a macrotheory of human motivation, development and health. Canadian Psychology, 49(3), 182-85. doi:10.1037/a0012801

23. González, L., Castillo, I., García-Merita, M., y Balaguer, I. (2015). Apoyo a la autonomía, satisfacción de las necesidades psicológicas y bienestar: invarianza de un modelo estructural en futbolistas y bailarines. Revista de Psicología del Deporte, 24(1), 121-129. Disponible en: https://www.rpd-online.com/article/view/1600

24. González, L., Tomás, I., Castillo, I., Duda, J. L., y Balaguer, I. (2016). A test of basic psychological needs theory in young soccer players: time-lagged design at the individual and team levels. Scandinavian Journal of Medicine and Science in Sports, 27(11), 1511-1522. doi:10.1111/sms. 12778

25. Graham, J. W., y Hofer, S. M. (2000). Multiple imputation in multivariate research. En T. D. Little, K. U. Schnable \& J. Baumert (Eds), Modeling longitudinal and multilevel data: Practical issues, applied approaches, and specific examples (pp. 201-218). Mahwah, NJ: Erlbaum.

26. Granero-Gallegos, A., Baena-Extremera, A., Sánchez-Fuentes, J. A., y Martínez-Molina, M. (2014). Perfiles motivacionales de apoyo a la autonomía, autodeterminación, satisfacción, importancia de la educación física e intención de práctica física en el tiempo libre. Cuadernos de Psicología del Deporte, 14(2), 59-70. Disponible en:

https://digitum.um.es/xmlui/bitstream/10201/402 30/1/199501-717271-1-SM.pdf

27. Kasser, T. (2002). Sketches for a selfdetermination theory of values. En E. L. Deci y R. M. Ryan (Eds.), Handbook of selfdetermination research (pp. 123-140). Rochester, NY: University of Rochester Press.

28. Lau, R. S., y Cheung, G. W. (2012). Estimating and comparing specific mediation effects in complex latent variable models. Organizational Research Methods, 15(1), 3-16. doi:10.1177/1094428110391673

29. Lonsdale, C., Hodge, K., y Rose, E. A. (2008). The Behavioral Regulation in Sport Questionnaire (BRSQ): Instrument development and initial validity evidence. Journal of Sport and Exercise Psychology, 30, 323-355. doi:10.1123/jsep.30.3.323

30. López-Walle, J., Balaguer, I., Castillo, I., y Tristán, J. (2011). Clima motivacional percibido, motivación autodeterminada y autoestima en jóvenes deportistas mexicanos. Revista de Psicología del Deporte, 20(1), 209-222. Disponible en: https://www.rpdonline.com/article/view/810

31. López-Walle, J., Balaguer, I., Castillo, I., y Tristán, J. (2012). Autonomy support, basic 
pychological needs and well-being in Mexican athletes. The Spanish Journal of Psychology, 15(03), 1283-1292. Disponible en: https://core.ac.uk/download/pdf/158457880.pdf

32. Ntoumanis, N. (2001). A self-determination approach to the understanding of motivation in physical education. British Journal of Educational Psychology, 71, 225-242. Disponible en: http://selfdeterminationtheory.org/SDT/document s/2001_Ntoumanis_BJEP.pdf

33. Oishi, S., Diener, E., Suh, E., y Lucas, R. E. (1999). Value as a moderator in subjective wellbeing. Journal of Personality, 24, 1319-1331. doi:10.1111/1467-6494.00051

34. Pineda-Espejel, H. A., López-Walle, J., y Tomás, I. (2015). Factores situacionales y disposicionales como predictores de la ansiedad y autoconfianza precompetitiva en deportistas universitarios. Cuadernos de Psicología del Deporte, 15, 55-70. doi:10.4321/S1578-84232015000200007

35. Rokeach, M. (1973). The nature of human values. New York, NY: Free Press.

36. Ryan, R. M., y Deci, E. L. (2000). Selfdetermination theory and the facilitation of intrinsic motivation, social development, and well-being. American Psychologist, 55, 68-78. Disponible en: https://selfdeterminationtheory.org/SDT/docume nts/2000_RyanDeci_SDT.pdf

37. Ryan, R. M., y Deci, E. L. (2001). On happiness and human potentials: a review of research on hedonic and eudaimonic well-being. Annual Review of Psychology, 52, 141-166. doi:10.1146/annurev.psych.52.1.141

38. Ryan, R. M., y Deci, E. L. (2002). Overview of self-determination theory: an organismic dialectical perspective. En E. L. Deci y R. M. Ryan (Eds.), Handbook of self-determination research (pp. 3-33). Rochester, NY: University of Rochester Press.

39. Ryan, R. M., y Deci, E. L. (2017). Selfdetermination theory: Basic psychological needs in motivation, development, and wellness. New York, NY: Guilford Press.
40. Ryan, R. M., y Frederick, C. (1997). On energy, personality, and health: Subjective vitality as a dynamic reflection of well-being. Journal of Personality, 65, 529-565. Disponible en: https://pdfs.semanticscholar.org/cb72/b5bc2b1e5 aefacbd191c7e0676d0c34b38bf.pdf

41. Ryan, R. M., Sheldon, K. M., Kasser, T., y Deci, E. L. (1996). All goals are not created equal: an organismic perspective on the nature of goals and their regulation. En P. M. Gollwitzer y J. A. Bargh. (Eds.), The psychology of action: linking cognition and motivation to behavior. New York, NY: Guilford Press.

42. Sagiv, L., y Schwartz, S. H. (2000). Value priorities and subjective well-being: Direct relations and congruity effects. European Journal of Social Psychology, 30, 177-198. doi:10.1002/(SICI)10990992(200003/04)30:2<177::AIDEJSP982>3.0.CO;2-Z

43. Schwartz, S. H. (1992). Universal in the content and structure of values: Theoretical advances and empirical tests in 20 countries. En M. P. Zanna (Ed.), Advances in experimental social psychology (Vol. 25, pp. 1-65). New York, NY: Academic Press. doi: 10138/235327

44. Schwartz, S. H., y Sortheix, F. M. (2018). Values and subjective well-being. En E. Diener, S. Oishi, y L. Tay (Eds.), Handbook of well-being. Salt Lake City, UT: DEF Publishers. Disponible en: http://hdl.handle.net/10138/235327

45. Schwartz, S. H., Cieciuch, J., Vecchione, M., Davidov, E., Fischer, R., Beierlein, C., Ramos,... Konty, M. (2012). Refining the theory of basic individual values. Journal of Personality and Social Psychology, 103, 663-688. doi:10.1037/a0029393

46. Sortheix, F. M., y Lönnqvist, J. E. (2015). Person- group value congruence and subjective well- being in students from Argentina, Bulgaria and Finland: The role of interpersonal relationships. Journal of Community \& Applied Social Psychology, 25(1), 34-48. doi:10.1002/casp.2193

47. Sortheix, F. M., y Schwartz, S. H. (2017). Values that underlie and undermine well-being: Variability across countries. European Journal of 


\section{Valores, motivación, bienestar e intención futura en baloncesto formación}

Personality,

$31(2)$

$187-201$ doi:10.1002/per.2096

48. Vallerand, R. J. (1997). Toward a hierarchical model of intrinsic and extrinsic motivation. En M. P. Zanna (Ed.), Advances in experimental social psychology (Vol. 29, pp. 271-360). New York, NY: Academic Press.

49. Viladrich, C., Appleton, P. R., Quested, E., Duda, J. L., Alcaraz, S., Heuze, J-P.,... Ntoumanis, N. (2013). Measurement invariance of the Behavioural Regulation in Sport Questionnaire when completed by young athletes across five European countries. International Journal of Sport and Exercise Psychology, 11, 384-394. doi:10.1080/1612197X.2013.830434.
50. Watson, D., Clark, L. A., y Tellegen, A. (1988). Development and validation of brief measures of positive and negative affect: The PANAS scales. Journal of Personality and Social Psychology, 54, 1063-1070. doi:10.1177/1073191108328890

51. Whitehead, J., Telfer, H., y Lambert, J. (2013). Values in youth sport and physical education. London: Routledge. doi: $10.4324 / 9780203114155$

52. World Health Organization (WHO, 2011). Promoting sport and enhancing health in European Union countries: A policy content to support action. Copenhagen (Denmark): Publication WHO Regional Office for Europe. 
Adell, F. L., Castillo, I., Álvarez, O., Tomás, I.

Tabla 3. Correlaciones bivariadas entre las variables del estudio

\begin{tabular}{lccccccccc}
\hline \multicolumn{1}{c}{} & 1 & 2 & 3 & 4 & 5 & 6 & 7 & 8 & 9 \\
\hline 1. Auto-trascendencia & - & & & & & & & & \\
2. Conservación & $-.23^{* *}$ & - & & & & & & \\
3. Autopromoción & $-.64^{* *}$ & $-.37^{* *}$ & - & & & & & \\
4. Apertura al cambio & .01 & $-.60^{* *}$ & -.12 & - & & & & \\
5. Motivación autónoma & .10 & .11 & $-.22^{*}$ & .03 & - & & & \\
6. Motivación controlada & $-.28^{* *}$ & .05 & $.25^{* *}$ & -.08 & .01 & - & & \\
7. No motivación & $-.22^{*}$ & .03 & $.26^{* *}$ & -.13 & $-.41^{* *}$ & $.60^{* *}$ & - & \\
8. Vitalidad subjetiva & -.04 & $.20^{*}$ & $-.28^{* *}$ & .06 & $.56^{* *}$ & -.18 & $-.43^{* *}$ & - \\
9. Afectos positivos & .01 & .18 & -.16 & -.04 & $.43^{* *}$ & .02 & $-.19^{*}$ & $.53^{* *}$ & - \\
10. Intención práctica futura & $.18^{*}$ & -.03 & $-.22^{*}$ & .13 & $.43^{* *}$ & -.14 & $-.39^{* *}$ & $.31^{* *}$ & $.37^{* *}$ \\
\hline
\end{tabular}

Nota. Para los cuatro valores se utilizaron las respuestas centradas. ${ }^{*} p<.01 ; * p<.05$

Tabla 5. Resultados de los efectos de mediación de los tipos de motivación en la relación entre los valores y los indicadores de bienestar así como de la intención futura de práctica

\begin{tabular}{|c|c|c|c|c|c|c|c|c|c|}
\hline Modelos & MA & $\mathrm{MC}$ & NM & VIT & AF-P & INT & $\begin{array}{c}\text { EI } \\
\text { VIT }\end{array}$ & $\begin{array}{c}\text { EI } \\
\text { AF-P }\end{array}$ & $\begin{array}{c}\text { EI } \\
\text { INT }\end{array}$ \\
\hline M1. Auto-trascendencia & .12 & $-.49 * *$ & $-.51 *$ & & & & & & \\
\hline Mediador MA & & & & $1.07 * *$ & $.53 * *$ & $.52 * *$ & .12 & .06 & .06 \\
\hline Mediador MC & & & & -.09 & .04 & .02 & .05 & -.02 & -.01 \\
\hline Mediador NM & & & & $-.22 \dagger$ & -.04 & $-.23 *$ & .11 & .02 & .12 \\
\hline $\mathrm{R}^{2}$ & .01 & .08 & .05 & $.36 * *$ & $.19 * *$ & $.23^{* *}$ & & & \\
\hline M2. Conservación & .17 & .11 & .08 & & & & & & \\
\hline Mediador MA & & & & $1.07^{* *}$ & $.54 * *$ & $.51^{* *}$ & .19 & .09 & .09 \\
\hline Mediador MC & & & & -.09 & .05 & .03 & -.01 & .01 & .01 \\
\hline Mediador NM & & & & $-.22 \dagger$ & -.03 & $-.23 *$ & -.02 & -.01 & -.02 \\
\hline $\mathrm{R}^{2}$ & .02 & .01 & .01 & $.36 * *$ & $.19 * *$ & $.23 * *$ & & & \\
\hline M3. Autopromoción & $-.15 * *$ & $.23 * *$ & $.31 * *$ & & & & & & \\
\hline Mediador MA & & & & $1.07 * *$ & $.54 * *$ & $.51 * *$ & $-.16^{\mathrm{sig}}$ & $-.08^{\mathrm{sig}}$ & $-.07^{\mathrm{sig}}$ \\
\hline Mediador MC & & & & -.10 & .04 & .03 & -.02 & .01 & .01 \\
\hline Mediador NM & & & & $-.22 \dagger$ & -.04 & $-.23^{*}$ & -.07 & -.01 & -.08 \\
\hline $\mathrm{R}^{2}$ & .06 & .06 & .07 & $.36 * *$ & $.19 * *$ & $.23 * *$ & & & \\
\hline M4. Apertura al cambio & .05 & -.13 & -.30 & & & & & & \\
\hline Mediador MA & & & & $1.07 * *$ & $.53 * *$ & $.52 * *$ & .05 & .03 & .02 \\
\hline Mediador MC & & & & -.09 & .05 & .03 & .01 & -.01 & -.01 \\
\hline Mediador NM & & & & $-.22 \dagger$ & -.04 & $-.23^{*}$ & .07 & .01 & .07 \\
\hline $\mathrm{R}^{2}$ & .01 & .01 & .02 & $.36 * *$ & $.19 * *$ & $.23 * *$ & & & \\
\hline
\end{tabular}

Nota. Coeficientes no estandarizados. $M A=$ Motivación autónoma; $M C=$ Motivación controlada. $N M=N o$ motivación; VIT = Vitalidad; AF-P = Afectos positivos; INT = Intención futura de práctica; $E I=$ Efecto indirecto. Para los valores se utilizaron las respuestas centradas. ${ }^{*} p<.01,{ }^{*} p<.05, t p<0.10$, sig $=$ efecto indirecto significativo (el intervalo de confianza no incluye el cero). 